\title{
Therapeutic drug safety for Indigenous Australians: how do we close the gap?
}

\author{
In the setting of significant disease burden, lack of data on drug safety for Australia's Indigenous \\ population is concerning
}

\begin{abstract}
ndigenous Australians have a high burden of disease and are at increased risk of premature death compared with the general Australian population.

Cardiovascular disease accounts for a large proportion of this burden, with high prevalence of type 2 diabetes and chronic renal failure being underlying risk factors. ${ }^{1}$

Traditional cardiovascular risk calculators underestimate risk in Aboriginals and Torres Strait Islanders. For example, observed numbers of coronary events for Indigenous Australians who live in remote areas are 2.5 times higher than predicted using the Framingham risk calculator, and younger women in this population have 30 times the predicted rate of events. ${ }^{2}$ While this suggests that solely focusing on management of traditional risk factors may not be the complete answer, this high burden of disease in the Indigenous population can drive recommendations for early and extensive use of medicines.
\end{abstract}

National guidelines recommend using lower thresholds to screen for and manage cardiovascular risk factors such as hyperlipidaemia in the Aboriginal and Torres Strait Islander population. ${ }^{3}$ Aggressive primary preventive measures for asymptomatic and young people, including early use of statin therapy, is recommended, despite limited direct evidence regarding efficacy in this population. It is also recognised that there are differences in the lipid profile of Indigenous Australians compared with non-Indigenous Australians, which may influence efficacy of therapies. ${ }^{4}$

Effective medications have adverse effects. Knowledge regarding drug safety is obtained across the lifecycle of drug use: throughout drug development programs, controlled clinical trials, and post-marketing experience. Drug development programs often do not include Indigenous Australians and few randomised controlled trials have been performed in this population. For many years (since the 1950s), the cornerstone of post-marketing safety has been spontaneously generated reports of adverse drug reactions from health care professionals and consumers. More recently, there has been an increasing focus on proactively investigating drug safety and using risk management plans in the post-marketing phase, rather than simply relying on spontaneously generated reports. Drug sponsors are required to report postmarketing adverse drug events to the Therapeutic Goods Administration (TGA), and certain groups are recognised as requiring special mention and consideration as they are often missing from clinical trials. ${ }^{5}$ Attention is given to reporting the safety of medications in older people and paediatric populations, and in pregnant and
Tilenka Thynne MBBS, FRACP ${ }^{1,2}$

Genevieve M Gabb MBBS(Hons), FRACP, GradDipClinEpi,

1 Flinders Medical Centre, Adelaide, SA.

2 Flinders University, Adelaide, SA.

3 Royal Adelaide Hospital, Adelaide, SA.

4 University of Adelaide, Adelaide, SA.

tilenka.thynne@ sa.gov.au

doi: 10.5694/mja15.00937 breastfeeding women. However, in Australia, there are no specific reporting requirements for ethnic groups, including Aboriginals and Torres Strait Islanders.

The Australian spontaneous adverse drug reporting system has only included a field to indicate racial status as "ATSI" (on the electronic form, not the paper form) since 2008, but this is not reliably completed (TGA, personal communication, 2013). Risk management plans are generally developed for specific products, rather than specific at-risk populations. Therefore, drug safety in Aboriginals and Torres Strait Islanders does not have a robust evidence base. The potential for harm is real.

In the face of this substantial uncertainty, several things are known.

First, the Australian Indigenous population, while ancient in tradition and culture, is a relatively youthful population in 2015. In 2011, the median age was 21.8 years, compared with 37.6 years for the nonIndigenous population. ${ }^{1}$ The significance of this is that a younger group of people are exposed to drugs when starting cardiovascular screening and primary preventive treatment, leading to potentially longer cumulative lifetime exposure.

Second, differences in drug response - efficacy and harm - exist in racially and ethnically distinct groups. ${ }^{6}$

Aboriginals and Torres Strait Islanders are a very diverse population, with over 250 different language groups, ${ }^{2}$ and it may be inappropriate to generalise what little information we do have with regard to efficacy and safety for the group as a whole. The causes of racial and ethnic differences in drug responses may be multifactorial. Potential intrinsic differences (eg, variation in genetics, metabolism and elimination) and extrinsic factors (eg, diet, environmental exposure and sociocultural factors) may play a role. In cardiovascular medicine, it has been established that African Americans respond poorly to $\beta$ blockers and angiotensin-converting enzyme (ACE) inhibitors, 7,8 and ACE inhibitor-associated angioedema is more prevalent (and possibly more severe) in African Americans than in white people. ${ }^{9}$ Racial and genetic variation contributes to variation in susceptibility to statin-associated myopathy, ${ }^{10}$ so lower initial statin drug doses are suggested for patients with Asian ancestry.

In the past decade, pharmacogenomics has advanced significantly, and moved from an individual candidate gene approach to the use of genome-wide association 
studies - that is, studies that compare the genomes of those affected by a disorder or drug-induced adverse effect to the genomes of those who are unaffected. Genome-wide association studies have consistently identified common variants in SLCO1B1 (a gene encoding the organic anion-transporting polypeptide OATP1B1, which regulates hepatic uptake of statins) that are strongly associated with an increased risk of statininduced myopathy. ${ }^{11}$ The prospective use of genotyping can help avoid adverse pharmacogenomic effects. Genotyping can also prevent classification of whole populations as diverse ethnic groups that are at universally high risk. For example, the Han Chinese have been identified as being at higher risk of Stevens-Johnson syndrome and associated severe toxic epidermal necrolysis when treated with carbamazepine. However, the discovery that HLA-B ${ }^{*} 1502$ carriers are the at-risk group has enabled successful genotype screening and treatment of HLA-B*1502-negative Han Chinese without any instances of Stevens-Johnson syndrome or toxic epidermal necrolysis. ${ }^{12}$

\section{"it may be inappropriate to generalise what little information we do have with regard to efficacy and safety"}

Third, it is known that harm occurs. In recent years, evidence has emerged from case reports and case series regarding drug safety and adverse drug reactions in Indigenous Australians, and some very significant adverse events from use of marketed medications have been published. Indigenous Australians may be at higher risk of serious, and potentially fatal, statinassociated myotoxicity, ${ }^{13-15}$ particularly in the setting of vitamin D deficiency. ${ }^{14}$ Recently, three cases of ACE inhibitor-associated angioedema involving airway compromise in Aboriginal Australians were reported. ${ }^{16}$ A genetic predisposition to a specific adverse drug reaction in the Indigenous population has also been suggested; a shared HLA-B allele was identified in three unrelated Indigenous patients who had severe phenytoin hypersensitivity syndrome, two of whom died. $^{17}$

The paucity of data on potential adverse drug reactions in the setting of a marked disparity in health standards between Indigenous and non-Indigenous Australians is of great concern. It is time to close the gap in drug safety information for Indigenous Australians. How will this be achieved?
The challenge is made greater by having to carefully balance the significant need for drug safety data while not further disadvantaging Aboriginals and Torres Strait Islanders. It is unrealistic and probably unethical to demand that all drugs be tested in an Aboriginal and Torres Strait Islander population before TGA approval. The financial costs alone would be prohibitive and this would significantly delay the introduction of new medications into the Australian market. This would also place a disproportionate burden on a minority group in terms of drug trial participation.

It is in the post-marketing space that a comprehensive and proactive approach to addressing drug safety in Indigenous Australians is urgently needed. The community as a whole - including health care providers, professional organisations, patients and regulators needs to recognise the significant lack of drug safety data available for Indigenous Australians and actively participate in promoting safety, including adverse event reporting.

All policies and guidelines promoting the quality use of medicine in the Aboriginal and Torres Strait Islander population must include a robust pharmacovigilance strategy and an acknowledgement of the limitations of drug safety information in this population. The assessment and management of potential adverse drug reactions should be part of any comprehensive health care program. Aboriginal health care workers, like all health care professionals, need training in pharmacovigilance, the principles of drug safety, and the identification and reporting of adverse drug reactions. This should be accompanied by culturally appropriate resources and tools to help them and their patients identify and manage adverse drug reactions. When adverse drug reactions do occur, these should be thoroughly investigated. ${ }^{18}$

Advances in other industries, typically aviation, have come from engaging with failures and investigating and reviewing bad outcomes. ${ }^{19}$

The same can be said of drug safety for Aboriginals and Torres Strait Islanders as for pharmacovigilance in general; it is not enough to be "content with an absence of evidence on harms ... we need to move to a position where we have evidence of absence of harm" ${ }^{20}$

Competing interests: No relevant disclosures.

Provenance: Not commissioned; externally peer reviewed.

(c) 2016 AMPCo Pty Ltd. Produced with Elsevier B.V. All rights reserved.

References are available online at www.mja.com.au. 
1 Australian Institute of Health and Welfare. The health and welfare of Australia's Aboriginal and Torres Strait Islander peoples: 2015. Canberra: AlHW, 2015. (AlHW Cat. No. IHW 147.) http://www.aihw.gov.au/publication-detail/?id=60129550168 (accessed Jul 2015).

2 Wang Z, Hoy WE. Is the Framingham coronary heart disease absolute risk function applicable to Aboriginal people? Med J Aust 2005; 182: 66-69. https://www.mja.com.au/journal/ 2005/182/2/framingham-coronary-heart-disease-absoluterisk-function-applicable-aboriginal

3 National Vascular Disease Prevention Alliance. Guidelines for the management of absolute cardiovascular disease risk 2012. National Vascular Disease Prevention Alliance, 2012. https:// strokefoundation.com.au/ /media/strokewebsite/resources/ treatment/absolutecvd_gl_webready.ashx?la=en (accessed Nov 2015).

4 O'Neal DN, Piers LS, Iser DM, et al. Australian Aboriginal people and Torres Strait Islanders have an atherogenic lipid profile that is characterised by low HDL-cholesterol level and small LDL particles. Atherosclerosis 2008; 201: 368-377.

5 Therapeutic Goods Administration. Australian requirements and recommendations for pharmacovigilance responsibilities of sponsors of medicines: Version 1.3. Canberra: TGA, 2014. https://www.tga.gov.au/book/2-reporting-requirements (accessed Jul 2015).

6 Ramamoorthy A, Pacanowski MA, Bull J, et al. Racial/ ethnic differences in drug disposition and response: review of recently approved drugs. Clin Pharmacol Ther 2015; 97: 263-273.

7 Rossi S, editor. Australian medicines handbook. Adelaide: $\mathrm{AMH}, 2015$.

8 Yancy CW, Fowler MB, Colucci WS, et al. Race and the response to adrenergic blockade with carvedilol in patients with chronic heart failure. N Engl J Med 2001; 344: 1358-1365.

9 Weber MA, Messerli FH. Angiotensin-converting enzyme inhibitors and angioedema: estimating the risk. Hypertension 2008; 51: 1465-1467.
10 Peters BJ, Klungel OH, Visseren FL, et al. Pharmacogenomic insights into treatment and management of statin-induced myopathy. Genome Med 2009; 1: 120.

1 Group SC, Link E, Parish S, et al. SLCOlBl variants and statin-induced myopathy-a genomewide study. N Engl J Med 2008; 359: 789-799.

12 Chen P, Lin JJ, Lu CS, et al. Carbamazepine-induced toxic effects and HLA-B*1502 screening in Taiwan. N Engl J Med 2011; 364: 1126-1133.

13 Gabb GM, Vitry A, Limaye V, et al. Serious statin-associated myotoxicity and rhabdomyolysis in Aboriginal and Torres Strait Islanders: a case series. Intern Med J 2013; 43: 987-992.

14 Haysom L, Samaras K, Stapylton C, et al. Statin-associated myotoxicity in an incarcerated Indigenous youth - the perfect storm. Med J Aust 2015; 202: 381-382. https://www.mja.com. au/journal/2015/202/7/statin-associated-myotoxicityincarcerated-indigenous-youth-perfect-storm

15 Wood J, Robertson T, Pui K et al. Statin-associated necrotizing autoimmune myopathies in the Indigenous population: a case series from North Queensland. Intern Med J 2015; 45(s20).

16 Mahajan H, Thynne T, Gabb GM, et al. Drug safety in Aboriginal Australians: three cases of angiotensin-converting enzyme inhibitor angioedema. Intern Med J 2015; 45(2): 231-233.

17 Harding DJ, Subramaniam K, MacQuillan G, et al. Severe drug-induced hypersensitivity syndrome with a shared HLA-B allele. Med J Aust 2012; 197: 411-413. https://www.mja.com.au/ journal/2012/197/7/severe-drug-induced-hypersensitivitysyndrome-shared-hla-b-allele

18 Carleton B, Poole R, Smith $\mathrm{M}$, et al. Adverse drug reaction active surveillance: developing a national network in Canada's children's hospitals. Pharmacoepidemiol Drug Saf 2009; 18: 713-721.

19 Syed M. Black box thinking: the surprising truth about success. London: John Murray General Publishing Division, 2015.

20 Evans S. Response to the Public consultation on legislative proposals for pharmacovigilance [letter]. European Commission, 2007. http://ec.europa.eu/health/files/ pharmacovigilance/docs/2007_02_26/19.pdf (accessed Jul 2015). ․ㅏ 